\title{
Evaporative Self-Assembly of Gold Nanorings via a Surface Acoustic Wave Atomization
}

\author{
Khee Chaw $\mathrm{Ng}^{\dagger}$, Aisha $\mathrm{Qi}^{\ddagger}$, Leslie Y. Yeo ${ }^{\ddagger}$, James Friend ${ }^{\ddagger}$ and Wenlong Cheng* ${ }^{\dagger}$ \\ ${ }^{\dagger}$ Department of Chemical Engineering, Monash University, Clayton, Victoria 3800, Australia \\ ${ }^{\ddagger}$ Department of Mechanical and Aerospace Engineering, Monash University, Clayton, Victoria 3800, \\ Australia \\ Email Correspondence: wenlong.cheng@monash.edu
}

\begin{abstract}
Ring of close packed gold nanoparticle arrays offers many fascinating properties that are not found in others assembly patterns. One of the most fantastic features of this unique organization is its ability to reroute shorter wavelengths of light in the visible region of electromagnetic spectrum, making it a very promising nanophotonic components for guiding light at the true nanoscale. Also, the creation of ring with gold nanoparticles can be used to make the world's smallest biosensors possible for multiple disease detection. Herein, we demonstrate a new paradigm for generating rings of CTAB-capped gold nanorods with the implementation of surface acoustic wave (SAW) atomization. With the ultrafast microfluidics actuation, the SAW atomizer can rapidly generate submicron fluids and efficiently form ring arrays onto desired substrates in less than 1s via the evaporative self-assembly process. The technique is able to provide a rational control over the of microfluids size distributions to engineer the smaller monodisperse rings arrays at micrometer scale. This microfluidics-assisted evaporative self assembly approach is also applicable to DNA-capped gold nanoparticles. The non-uniform mass distribution of ring is formed upon the pinning of contact line to substrates during a far-fromequilibrium dewetting process. Our method opens an avenue towards the ring assembly of gold nanoparticles in their ultimate microscopic minimal threshold to facilitate the generation of metamaterials.
\end{abstract}

Keywords: Gold nanorods, rings, microfluidics surface acoustic wave atomization

Smart Nano-Micro Materials and Devices, edited by Saulius Juodkazis, Min Gu, Proc. of SPIE

Vol. 8204, 820434 - (C) 2011 SPIE · CCC code: 0277-786X/11/\$18 · doi: 10.1117/12.903172

Proc. of SPIE Vol. $8204820434-1$ 


\section{Introduction}

Assembly of gold nanoparticles ${ }^{1,2}$ into ring-liked superstructures (gold nanorings) has gained widespread attention owing to their ability to localize, guide and enhance light in at true nanometer scale. For example, gold nanorings made of the plasmonic gold nanoparticles are able to reroute rays of visible light, making the superstructures a very efficient

nanophotonic component for light guiding ${ }^{3}$. Also, gold nanorings at their ultimate minimal size threshold can also be used to make the smallest biosensors ${ }^{4}$ for multiple disease detection. By packing thousands, even millions, of small micro-biosensors onto a lab-on-a-chip, this allows one to perform a large number of medical diagnostics on a single chip. In the past decades, several studies have reported the formation of micrometer rings made of nanocrystals ${ }^{5}$, polymer capped gold nanorods ${ }^{6}$ and Ni-coated gold nanorods ${ }^{7}$. However, the formations often involve the direct self-assembly of evaporating nanoparticles droplets on a solid substrate which are basically induced by various mechnisms that related to Marangoni effect, such as crystal growth, paint film drying, wetting processes, thin films physics or external magnetic field. To the best of our knowledge, there is no report on rings assembly with microfluidic based technique so far. In this work, we demonstrate the assembly of hexadecyltrimethylammonium bromide (CTAB)-capped gold nanorods (GNRs) and DNA-capped gold nanoparticles into nanorings superstructure using a surface acoustic wave (SAW) atomization technique ${ }^{8,9}$. SAW atomization is a microfluidic technique originally developed for different purposes of signal filtering and multicomplexing ${ }^{10}$. It provides direct control over the droplet size with varying operating frequency of ultrasonic vibration through the design of electrode design ${ }^{11}$. With its high operating frequencies, the SAW atomization was employed here to break up a large droplet into in micro-sized droplet within a narrow size range $(1-10 \mu \mathrm{m})$ for instant small ring assembly onto desired substrates.

\section{Experimental Details}

\subsection{SAW Devices}

The SAW device consisted of a single interdigital transducer (IDT) with 25 pairs of 400nm thick, straight aluminium/titanium electrodes sputter-deposited onto a piezoelectric substrate via UV-photolitography (Fig. 1). The piezoelectric substrate was made of a single crystal 127.68 yx-cut lithium niobate $\left(\mathrm{LiNbO}_{3}\right)$. To atomize the GNRs solution into $1-10 \mu \mathrm{m}$ micro-sized droplets, the operating frequency was set at $29.73 \mathrm{MHz}$. Using an RF signal generator and RF power amplifier, a sinusoidal electrical signal matching the operating frequency i.e. a working voltage of $250 \mathrm{mV}$ was applied to the IDT to generate the SAW. Two displacement modes were possessed when the SAW travelling along the surface of substrate: a longitudinal component along the direction of propagation and a transverse component perpendicular to the surface. At low amplitude and power, the longitudinal mode was diffracted into the GNRs droplet 
at Rayleigh angle,$\theta_{\mathrm{R}}=\sin ^{-1}\left(\mathrm{c}_{1} / \mathrm{c}_{\mathrm{s}}\right)$ due to the mismatch of bulk wave velocity in solid, $\mathrm{c}_{\mathrm{s}}$ and in liquid, $\mathrm{c}_{1}$ at the interfaces $^{12-14}$. By slowly increasing the power, capillary waves were induced by the leaky SAW acoustic field and the capillary stress at the interfaces was overcome, leading to an interfacial destabilization that causes the solution to break up into submicron-sized droplets.

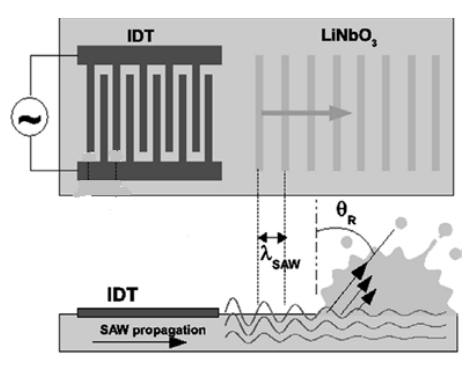

Figure 1 Schematic of Rayleigh wave propagation and the atomization process ${ }^{8}$.

\subsection{Gold Nanoparticles Preparation}

\subsubsection{Gold nanorods Synthesis}

A well-established seed-mediated growth method was used to synthesize gold nanorods ${ }^{2}$. In a typical way, the seed solution was prepared by stir-mixing CTAB $(5.0 \mathrm{ml}, 0.2 \mathrm{M})$ and gold (III) chloride trihydrate, $\mathrm{HAuCl}_{4}(5.0 \mathrm{ml}, 0.5 \mathrm{mM})$ at low speed. Addition of ice cold $\mathrm{NaBH}_{4}(0.6 \mathrm{ml}, 0.01 \mathrm{M})$ into the mixture, followed by a 3min vigorous stirring changed the solution color from bright to brownish yellow. The seed solution was then aged at $25^{\circ} \mathrm{C}$ before seeding to growth solution. In the preparation of growth solution, $0.15 \mathrm{ml}$ of $4 \mathrm{mM} \mathrm{AgNO}_{3}$ was mixed with a CTAB solution (5.0ml, $0.2 \mathrm{M}$ ), followed by $\mathrm{HAuCl}_{4}(5.0 \mathrm{ml}, 1.0 \mathrm{mM})$. The yellowish mixture turned colorless with the addition of ascorbic acid $(0.07 \mathrm{ml}$, $0.0788 \mathrm{M})$. Upon rods growth, $12 \mathrm{uL}$ of premade seed was added into the growth solution. The solution was aged at a constant temperature of $30^{\circ} \mathrm{C}$ for 2hours to ensure the production of GNRs in the solution. Before the atomization, the GNRs solution was purified by 3 times centrifugation (10min, 10krpm) to remove unreacted chemicals. The mean aspect ratio and concentration of the nanorods were estimated through their correlations to plasmon band extinction coefficient ${ }^{15}$.

\subsubsection{DNA-capped Gold Nanoparticles Synthesis}

Gold nanoparticles with diameters of $13 \mathrm{~nm}$ were synthesized following to the literature ${ }^{1}$. Typically, a auric solution were prepared by diluting $2 \mathrm{~mL}$ HAuCl 4 solution $\left(25 \mathrm{mM}\right.$ ) with $170 \mathrm{~mL}$ Milli-Q water, followed by heating to $100^{\circ} \mathrm{C}$ until boiling. Then, $6 \mathrm{~mL}$ sodium citrate solution (34 mM) was added into the above auric solution. After heating for 3 min, the clear solution turned into a wine-red color, which indicated the formation of Au nanoparticles. The heat source 
was removed after further boiling for 10 minutes and the dispersion was cooled down to room temperature before capping with DNA. ssDNA-capped nanoparticles were prepared according to a protocol similar to Mirkin and coworkers' report ${ }^{16}$. 50-thiolated ssDNA (Integrated DNA Technologies) was deprotected and incubated with nanoparticle solutions with a DNA/nanoparticle ratio of 1000:1. The mixture was then allowed to stand for $12 \mathrm{~h}$ at room temperature, after which sodium chloride was added to a final concentration of $1 \mathrm{M}$. Finally, the mixture was aged at room temperature for another 10 to12 $\mathrm{h}$ and centrifuged and exchanged in Milli-Q water.

\subsection{Gold Nanorings Assembly Setup, Procedure and Characterization}

The experimental setup consisted of a pipette tip, connected one side to the SAW device and another side to a vacuum pump with parafilm (Fig.2). A $1 \mathrm{~cm} \times 0.1 \mathrm{~cm}$ rectangular hole was drilled on the surface of pipette tip to hold solid substrates (TEM grids or SEM silicon wafer) approximately 2cm above the SAW device. The TEM grid was hold by a tweezer and the silicon wafer was intentionally cut into a dimension slightly bigger than the hole i.e. $1 \mathrm{~cm} x 1 \mathrm{~cm}$ so that it could stand by itself on the hole without any support. To ensure the evaporative micro-droplets (i.e. atomized by the SAW device) were easily extracted onto solid substrates, the vacuum pump was used to introduce a directed atomization path during each atomization.

The SAW atomization procedure for ring assembly was as follows: Firstly, an operating frequency of $29.73 \mathrm{MHz}$ and a working voltage of $250 \mathrm{mV}$ were set. Secondly, the RF amplifier was tuned to an optimum magnitude for homogeneous atomization. Then, $0.5 \mathrm{ul}$ of nanoparticle solution was placed at the center of lithium niobate i.e. to avoid the entire droplet moves across the substrate. Finally, the solid substrate was placed in the pipette tip hole and the vacuum pump was turned on before siwtching on the RF signal generator. The evaporative self-assembled gold nanoparticles via SAW atomization collected on the silicon wafer and TEM copper grids were characterized with Jeol -7001F and Hitachi H7500 field emission TEM operating at $80 \mathrm{kV}$ respectively. Through proper washing the SAW device with mili-Q water and nitrogen drying, the device was reusable for each atomization.

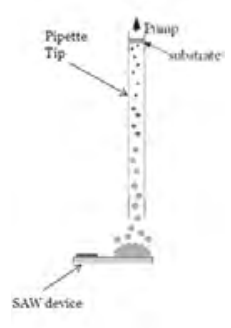

Figure 2 Schematic of SAW atomization for nanorings assembly setup. 


\section{Results and Discussion}

We began the self assembly of gold nanorods through SAW atomization with high concentration GNRs solutions. At GNRs concentrations from $43 \mathrm{nM}$ to $70 \mathrm{nM}$, the atomization instantly generates submicron ring-liked assemblies on the TEM substrates in less than one second (Fig.3a). For each individual nanoring, the edge contains much higher rods concentration than the middle, forming ring with darker rim. The size of nanoring is rationally small, approximately 8.9 $\pm 0.2 \mu \mathrm{m}$ in diameter (Fig.3b). Conversely, no ring-liked formations were produced upon SAW atomization of gold nanorods solution with reduced nanoparticles concentrations i.e. 10nM to 35nM. At this low concentration regime, the nanorods self-assembled as islands of agglomerates, uniformly dispersing throughout the nanostructures (Fig.3c). Similar kind of uniform agglomerates were also observed when placing a small piece of kim wipes fibre on the piezoelectric substrate to generate continuous atomization (Fig.3d). This is due to a massive nanorods loss as a result of strong fluid absorption nature of kim wipe that reduced the nanorods concentration upon ring assembly. The agglomerates formation at low nanorods concentration reveals that the ring assembly will only occur at a concentration above certain threshold. In this case, our ring of nanorods assembly was achieved at a concentration threshold of 43nM.

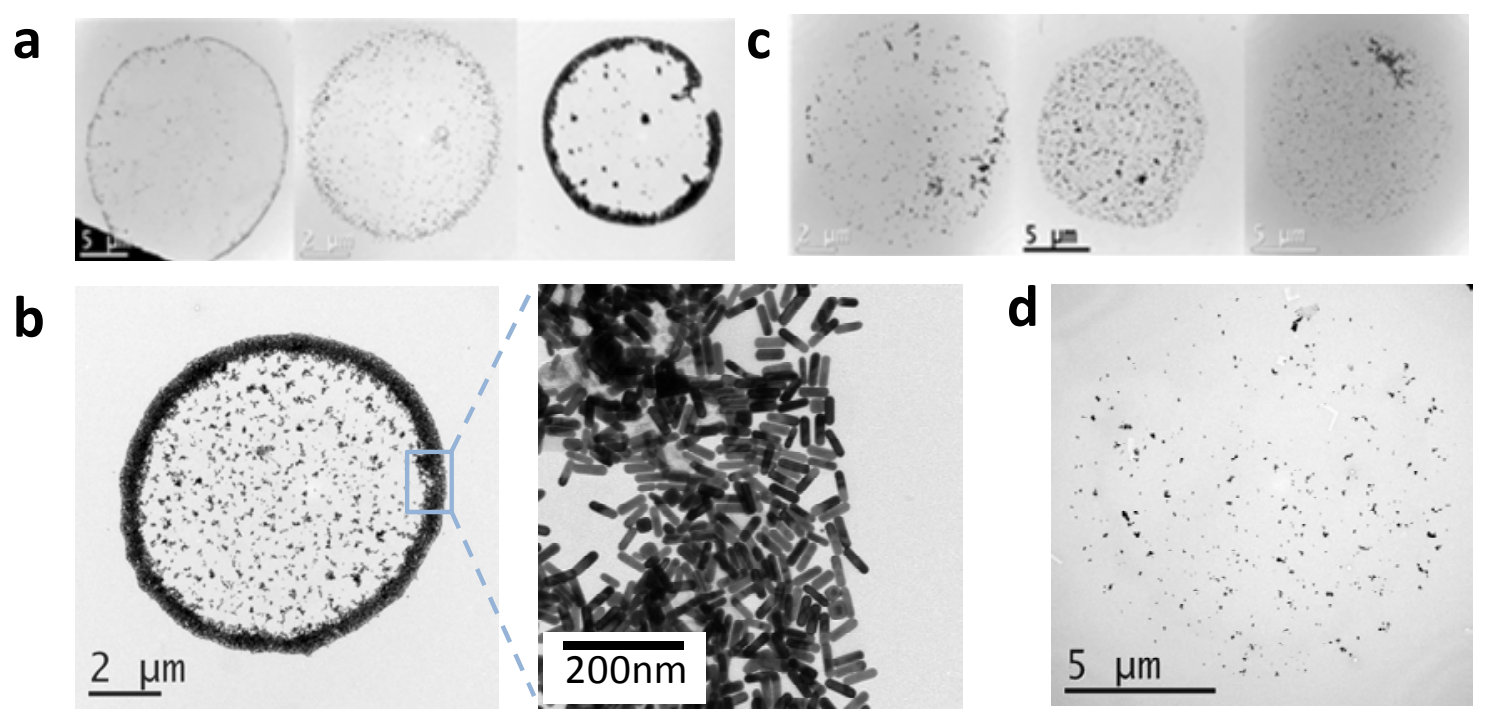

Figure 3 (a) TEM images of gold nanorings formed via SAW atomization of gold nanorods solution at 45nM, 59nM, 70nM (left to right). (b) TEM image of ring formed at $66 \mathrm{nM}$. The magnified section of (d) shows the gold nanorods are concentrated at the rim of the ring. (c) TEM images of agglomerates formed via SAW atomization of gold nanorods solution at 10nM, 27nM, 35nM (left to 
right). (d) TEM image of agglomerates formed as a result of material loss through absorption of kim wipes fibre during the SAW atomization.

Ring formation is a coffee ring phenomenon that commonly occurs in our daily life. Very often, spillage of coffee droplet on a table would always leave behind a ring-liked stain along the perimeter after liquid evaporation. In fact, these phenomena can occur in a wide variety of substrates, solutes and solvents as long as the solvent meets the surface at a non-zero contact angle, the contact line is pinned to its initial position and the solvent evaporates. The solute transportsurface tension gradients, solute diffusion, electrostatic and gravity effects are negligible in the ring formation ${ }^{17}$. Similarly in our gold nanoring assembly, the non-uniform mass distribution of rings was induced by the coffee ring phenomenon which was mainly constrained by pinned contact line that squeezes the fluid outward from the interior to compensate the liquid evaporation losses at the edge during the dewetting process. As the atomized micro-droplets evaporated on the substrate, nanorods suspended inside the liquid tend to transport outward by water flow to the microdroplet's perimeter. At the micro-droplet edge, the nanorods became increasingly concentrated as evaporation proceeding. Eventually upon fully water evaporation, the nanorods were concentrated around the rim to form a ring with darker rim.

Interestingly, we observed some monodisperse GNRs sheet other than gold nanoring formations via SAW atomization of nanorods (above threshold concentration). The diameter of gold nanoring is noticeably much larger than the small monodisperse sheet. Based on Figure 4, the diameters of the gold nanorings, $d_{\text {ring }}$ and the monodisperse sheet, $d_{\text {sheet }}$ are $8.9 \pm 0.2 \mu \mathrm{m}$ and $0.78 \pm 0.08 \mu \mathrm{m}$ respectively, which implies that the ring formation is fairly sensitive to the size of atomized droplets. Through the evaporation of large atomized nanoparticles droplets, rings of rods were induced as the nanoparticles dispersed in the liquid have sufficient time to move to rim and concentrated around the ring before water fully evaporates. For smaller atomized droplets, water at the solid-liquid interfaces evaporates faster than the nanorods move from the center replacing the evaporated rim fluid, thereby having insufficient time to move to the rim and resulted in nanorods sheets. We further applied the microfluidics-assisted evaporative ring assembly approach towards DNAcapped gold nanoparticles. Again, those two nanostructures were formed and the ring of DNA-capped gold nanoparticles is still larger than the monodispersed sheet i.e. $d_{\text {ring }}=9.1 \pm 0.4 \mu \mathrm{m}, d_{\text {sheet }}=1.40 \pm 0.05 \mu \mathrm{m}$, which account for the droplet size dependency on the ring formation (Fig. 5). Evaporation of large atomized droplets favors the ring assembly. 

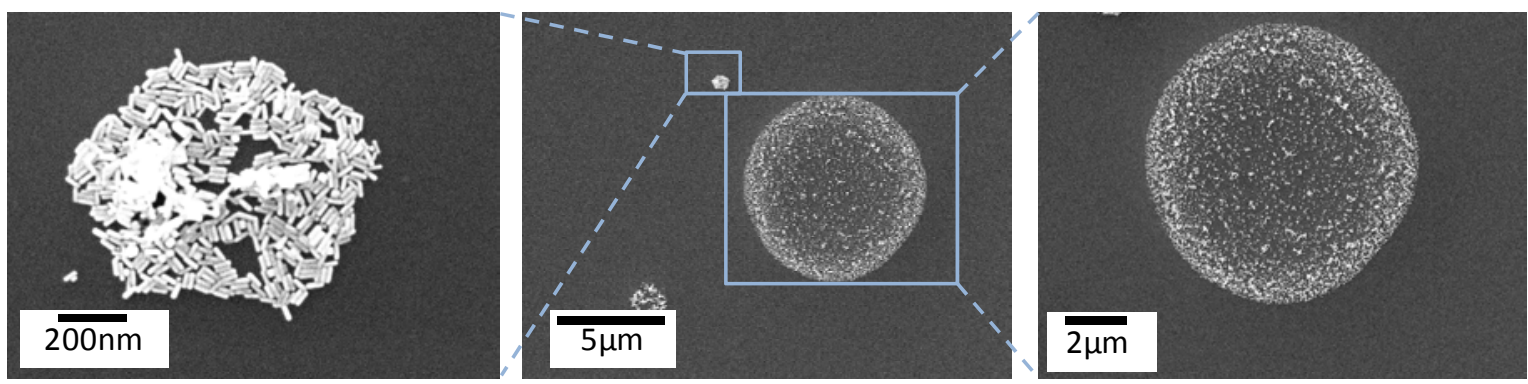

Figure 4 SEM image of gold nanoring and gold nanosheet formed via SAW atomization of GNRs solution at 66nM (middle).The ring-liked formation was achieved through atomizing larger droplet(right). Atomization of smaller droplet leads to the formation of nanosheet. The magnified section (left) reveals that the nanorods uniformly distributed throughout the entire sheet.
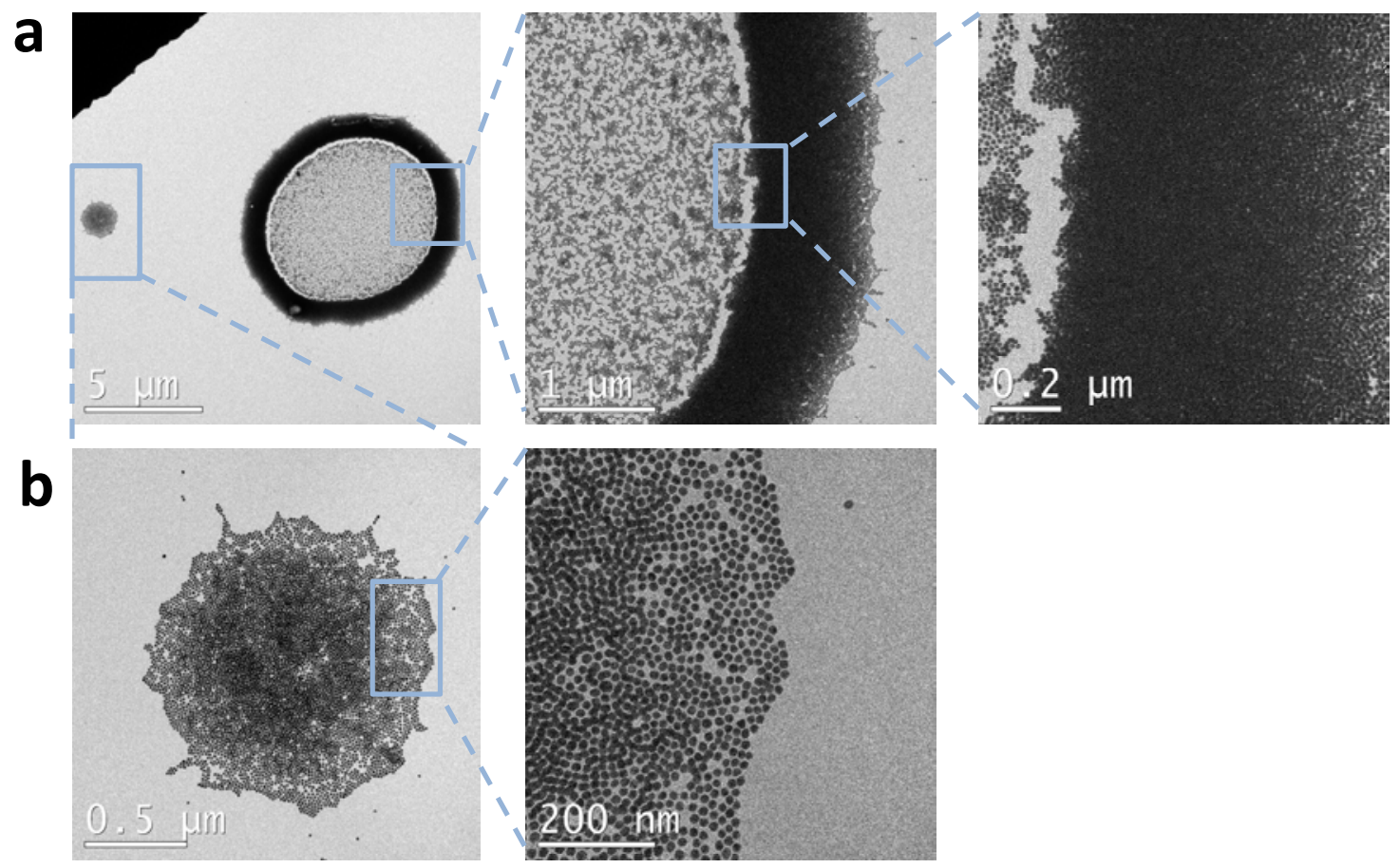

Fig. 5 TEM images of gold nanoring and gold nanosheet formed via SAW atomization of DNA-gold nanoparticles solution at 400nM (a) The ring-liked formation was induced through atomization of larger droplet. (b) Atomizating smaller droplet leads to the formation of nanosheet. The magnified section of (a) show the ring rim contains higher concentration of DNA-capped gold nanoparticles than the inner region. The magnified section of (b) reveals that the DNA-capped gold nanoparticles uniformly distributed throughout the entire sheet. 


\section{Conclusions}

In conjunction with the evaporative self assembly, SAW atomization generates rings of gold nanoparticles at micrometer scale and the rings could be collected on a variety of substrates. The ring formation was achieved at a concentration threshold of 43nM with a minimum atomized droplet of $8.9 \mu \mathrm{m}$. The ring growth was inhibited with small atomized droplets. Our microfluidic assisted ring-assembly approach potentially opens a new avenue towards the design of metamaterials for building nanophotonic components and tiny biosensors.

\section{Acknowledgements}

This work is funded by New Staff Member Research Fund (2437404), Faculty of Engineering and by Department of Chemical Engineering, Monash University. The authors would like to thank, Yi Chen, Yue Tang, Monash Micro Imaging Centre, The monash centre for Electron Microscopy, Joan Clark, Dr. Flame Burgmann, Dr. Xi Ya Fang for all the technical supports.

\section{References}

[1] Frens, G. "Controlled Nucleation for Regulation of Particle-Size in Monodisperse Gold Suspensions". NaturePhys Sci 241, 20-22 (1973).

[2] Nikoobakht, B. \& El-Sayed, M. A. "Preparation and growth mechanism of gold nanorods (NRs) using seedmediated growth method". Chem Mater 15, 1957-1962 (2003).

[3] Maier, S. A. et al. "Local detection of electromagnetic energy transport below the diffraction limit in metal nanoparticle plasmon waveguides". Nat Mater 2, 229-232 (2003).

[4] Li, J. F. et al. "Shell-isolated nanoparticle-enhanced Raman spectroscopy". Nature 464, 392-395 (2010).

[5] Maillard, M., Motte, L., Ngo, A. T. \& Pileni, M. P. "Rings and hexagons made of nanocrystals: A Marangoni effect". J Phys Chem B 104, 11871-11877 (2000).

[6] Khanal, B. P. \& Zubarev, E. R. "Rings of nanorods". Angew Chem Int Edit 46, 2195-2198 (2007).

[7] Ahmed, W. et al. "Dipole directed ring assembly of Ni-coated Au-nanorods". Chem Commun 46, 6711-6713 (2010). 
[8] Alvarez, M., Friend, J. \& Yeo, L. Y. "Rapid generation of protein aerosols and nanoparticles via surface acoustic wave atomization". Nanotechnology 19 (2008).

[9] Friend, J. R., Yeo, L. Y., Arifin, D. R. \& Mechler, A. "Evaporative self-assembly assisted synthesis of polymeric nanoparticles by surface acoustic wave atomization". Nanotechnology 19 (2008).

[10] White, R. M. \& Voltmer, F. W. "Direct Piezoelectric Coupling to Surface Elastic Waves". Appl Phys Lett 7, 314-\& (1965).

[11] Lang, R. J. "Ultrasonic Atomization of Liquids". J Acoust Soc Am 34, 6-\& (1962).

[12] Shiokawa, S., Matsui, Y. \& Ueda, T. "Study on Saw Streaming and Its Application to Fluid Devices". Jpn J Appl Phys 1 29, 137-139 (1990).

[13] Wixforth, A. et al. "Acoustic manipulation of small droplets". Anal Bioanal Chem 379, 982-991 (2004).

[14] Chono, K., Shimizu, N., Matsui, Y., Kondoh, J. \& Shiokawa, S. "Development of novel atomization system based on SAW streaming". Jpn J Appl Phys 1 43, 2987-2991 (2004).

[15] Orendorff, C. J. \& Murphy, C. J. "Quantitation of metal content in the silver-assisted growth of gold nanorods". J Phys Chem B 110, 3990-3994 (2006).

[16] Thaxton, C. S., Hill, H. D., Georganopoulou, D. G., Stoeva, S. I. \& Mirkin, C. A. "A bio-bar-code assay based upon dithiothreitol-induced oligonucleotide release". Anal Chem 77, 8174-8178 (2005).

[17] Deegan, R. D. et al. "Capillary flow as the cause of ring stains from dried liquid drops". Nature 389, 827-829 (1997). 